\title{
Dose Tapering of Biologics in Patients with Psoriasis: A Scoping Review
}

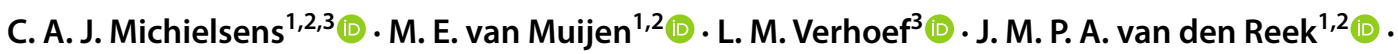 \\ E. M. G. J de Jong ${ }^{1,2,4}$
}

Published online: 16 January 2021

(c) The Author(s) 2021

\begin{abstract}
Introduction Biologics serve as a cornerstone in psoriasis treatment, with low disease activity or sometimes even clinical remission as a realistic treatment outcome. So far, it is unclear whether biologics should be tapered when this target is achieved. Dose tapering could offer potential benefits by decreasing side effects, the burden of repetitive injections and costs of biological therapy. However, clinical guidelines on dose tapering of biologicals in psoriasis patients are lacking. This scoping review was conducted to provide an overview of the current literature on dose tapering and offer guidance for clinicians in daily clinical practice.

Methods Dose tapering is defined as the administration of a lower dose per administration, or the prolongation of the regular dose interval, after initial treatment according to the standard dosing. Four electronic databases (PubMed, EMBASE, Cochrane, and Web of Science) were systematically searched for literature on tapering of biologics in adult patients with psoriasis from 1 January 2000.

Results We included 19 original articles on biologic tapering in psoriasis patients: four randomized controlled trials and 15 observational studies. Tapering eligibility criteria, tapering strategies, tapering outcomes, and recapture of response after relapse were assessed. Furthermore, the available evidence on possible predictors for successful tapering, and the effect of tapering on safety, quality of life and costs is summarized. The definition of low disease activity as a measure for tapering eligibility varied widely. Beside tapering criteria, tapering strategies were also heterogeneous. Of note, quality-of-life measurements were barely integrated in the evaluation of tapering outcomes. Literature on regaining response after relapse due to tapering was limited, but restored remission has been described. The included studies did not proclaim a significant effect of tapering on the occurrence of (severe) adverse events. Even though cost savings have been reported, no proper cost-effectiveness analysis has been conducted yet.

Conclusion Biologic tapering seems to be effective and safe in psoriasis patients with stable low disease activity or clinical remission. Available data on biologic dose tapering in patients with psoriasis are promising, but more research is warranted to fill the current gaps in knowledge.
\end{abstract}

\section{Plain Language Summary}

Biologics are effective in treating psoriasis amongst other diseases, such as rheumatoid arthritis and Crohn's disease. However, biologics are costly, and can cause side effects, such as an increased risk of infection. In patients with rheumatoid arthritis, it is not uncommon to lower the dose of these biologics (also called "dose tapering"), once stable low disease

C. A. J. Michielsens and M. E. van Muijen share first authorship.

Digital Features To view digital features for this article go to https://doi.org/10.6084/m9.figshare.13256477.

Supplementary Information The online version contains supplementary material available at https://doi.org/10.1007/s4026 5-020-01448-z.

C. A. J. Michielsens

Celia.Michielsens@ radboudumc.nl

Extended author information available on the last page of the article 
activity, or even remission, is reached. However, in psoriasis patients, dose tapering of biologics is not common practice. In this "scoping review," we provide an overview of the available literature on dose tapering of biologics in adult patients with plaque psoriasis in order to address the current gaps in literature. We found 19 studies that addressed dose tapering. These studies used different criteria to determine which patients were eligible for tapering, which led to various interpretations of tapering success. This made it difficult for us to draw general conclusions on which tapering criteria and strategies should be further investigated. Dose tapering seems to be effective and safe in patients with a stable low disease activity, although more (high-quality) research is needed. Future studies should focus on generating more data on long-term safety, finding predictors for successful tapering, calculating the cost-effectiveness of dose tapering, and evaluating dose tapering in the newest generation of biologics.

\section{Key Points}

Due to a variation in tapering strategies (including eligibility criteria, tapering regimens, and success criteria) among studies, success rates of dose tapering differ in the literature.

Dose tapering of biologic therapy in psoriasis patients with low disease activity or clinical remission seems effective and safe in a substantial number of patients.

Identified research gaps are: description of and usage of uniform tapering criteria and strategies, and the longterm impact of tapering on disease activity, quality of life, and safety/immunogenicity. Also, dose tapering of interleukin-17 and interleukin-23 inhibitors is an important future topic of interest.

\section{Introduction}

Psoriasis is an immune-mediated inflammatory skin disease characterized by different clinical phenotypes, with chronic plaque psoriasis accounting for $90 \%$ of cases. In addition to the skin disease, psoriasis is associated with co-morbidities such as psoriatic arthritis, which contributes to the impairment of quality of life (QoL) and physical functioning [1]. Biologic therapy has proven to be effective in controlling the inflammatory burden of this chronic skin disease, and its emerging co-morbidities. Despite its effectiveness, the use of biologics also comes with disadvantages like high costs [2] and adverse events (e.g., injection site reactions, adverse drug reactions, and infections) [3-7]. The question arises if these disadvantages can be diminished by biologic tapering, when a state of stable low disease activity or clinical remission is reached.

Most of the current evidence on biologic tapering concerns rheumatologic diseases, and tapering strategies have already been implemented in the European recommendations for rheumatoid arthritis (RA) [8]. In psoriasis, literature on treatment outcomes of dose tapering of biologics is scarce. Data on patients using reduced doses of biologics has mainly been described in (observational) studies, not specifically intending to evaluate the effect of dose tapering. However, even studies that are not primarily aimed to evaluate the effect of dose tapering can contribute to the body of evidence.

In this scoping review, our aim was to provide a broad overview of the available literature on dose tapering of biologics in adult patients with plaque psoriasis. We assessed tapering eligibility criteria, tapering strategies, and tapering outcomes, as well as safety, QoL, and cost aspects of tapering. We aimed to provide new insights valuable for implementation in clinical practice, and to identify gaps in the current evidence, providing insights for future research on dose tapering.

\section{Methods}

\subsection{Search Strategy}

Four electronic databases (PubMed, EMBASE, Cochrane, and Web of Science) were systematically searched for literature between 1 January 2000 and 10 April 2020. Literature was searched since 2000, as the first biologic for psoriasis treatment was US Food and Drug Administration (FDA) approved in 2003 [9, 10]. The search strategy combined terms on (1) psoriasis, (2) biologic therapies, and (3) verbs associated with dose reduction or tapering. All possibly relevant synonyms, hyponyms, and generic names were included. The search was limited to studies written in English, French, Dutch, and German. The full electronic search strategy is described in Online Resource 1 (see Online Supplemental Material, OSM). Selected articles were imported into EndNote X9 bibliographic management software. Reference lists of the included articles were screened for additional studies of interest. If necessary, corresponding authors were contacted to obtain more in-depth information. This scoping review was performed based on a predefined 
protocol. Registration in Prospero was not required as this is a scoping review.

\subsection{Inclusion and Exclusion Criteria}

All studies providing original data on dose tapering of biologic therapy in adults with psoriasis were included, but reviews, conference abstracts, and case reports were excluded. Dose tapering was defined as the administration of a lower dose per administration, or the prolongation of the regular dose interval, after initial treatment according to the registered dosing. In case of two registered doses (e.g., tildrakizumab $200 \mathrm{mg}$ and $100 \mathrm{mg}$ ), stepping down from a higher to a lower registered dose was not considered dose reduction, with the exception of weight-appropriate registrations (ustekinumab). Studies on biologic discontinuation, retreatment-as-needed strategies, updosing, and biologic therapies not currently registered for psoriasis were excluded, as well as studies in which biologic therapy was prescribed for indications other than plaque psoriasis.

\subsection{Study Selection}

Screening of titles and abstracts was performed by one reviewer (CM or MvM). Full articles were independently checked by two reviewers (CM and MvM) for inclusion and exclusion criteria. Discrepancies were resolved by a third reviewer (JvdR).

\subsection{Data Extraction and Outcome Measures}

Data charting was performed in duplicate by CM and MvM. If available, the following data were extracted in a predesigned charting form: study characteristics, funding, type of biologic therapy, number of patients, study inclusion- and exclusion criteria, tapering eligibility criteria, tapering strategy, treatment duration prior to tapering, retreatment strategy in case of relapse, study duration and treatment duration on a tapered dose, tapering outcomes, time to relapse, effectiveness after retreatment, adverse events, predictors for successful tapering, effect on QoL, costs, and other relevant issues addressed in the studies. Meta-analyses were not performed, as the aim was not to obtain summarized estimates for one or two outcomes, but to anticipate relevant issues raised when considering tapering of biologics in psoriasis in a scoping manner. All items of the Preferred Reporting Items for Systematic reviews and Meta-analyses extension for Scoping Reviews (PRISMA-ScR) checklist were addressed. In line with the usual methodology for scoping reviews, risk of bias was not performed [11].

\section{Results}

\subsection{Included Studies}

The search strategy yielded 4809 unique articles, of which 19 full-text articles were eligible according to our selection criteria (Fig. 1). Four randomized controlled trials (RCTs) and 15 observational studies were included. Among the four RCTs [12-15], Atalay et al. carried out an openlabel, non-inferiority RCT on adalimumab, etanercept, and ustekinumab to evaluate if a dose-reduction strategy was non-inferior to usual care [12]. Blauvelt et al. aimed to assess the prolongation of ustekinumab maintenance-dosing intervals in a phase IIIb, randomized, double-blinded, active treatment-controlled multicenter study [13]. Lebwohl et al. described the results of two phase III studies with an identical design, comparing various dosages of brodalumab to ustekinumab and placebo [14]. These studies had a multicenter, randomized, double-blinded, placebo-controlled and active comparator-controlled, parallel-group design, but were carried out to compare the efficacy and safety of brodalumab with ustekinumab, rather than to evaluate dose reduction. However, with brodalumab tapering performed in 1,698 patients, the study by Lebwohl et al. provides valuable information. In a randomized, open-label, rater-blinded phase IIIb study, Reich et al. compared secukinumab every4-week (Q4W) dosing to Q6W dosing in patients who had achieved a 90\% improvement in the Psoriasis Area and Severity Index (PASI90) after 24 weeks of standard dosing [15]. In the 15 observational studies, three studies had a prospective [16-18] and 12 studies a retrospective design [19-30]. Similar to the study by Lebwohl et al. [14], dose tapering was not the main objective of these observational studies. Within the 15 observational studies, five studies (two prospective [16, 18], three retrospective [20, 24, 27]) specifically aimed to evaluate the effect of dose tapering for different types of biologics. The ten other studies looked for polymorphisms associated with successful dose reduction [17], explored the frequency or patterns of dose adjustments $[19,21,22,25]$, evaluated the efficacy and safety of adalimumab treatment $[23,26]$, made an informal cost analysis [28], evaluated the effect of a pharmacotherapeutic protocol [29], or searched for clinical factors associated with dose reduction or escalation [30]. As for all studies included, only relevant study arms will be discussed in this review. For each included article, Table 1 depicts the study design, number of patients included, biologics that were tapered, and topics addressed. Table 2 provides an overview of the tapering criteria and strategies used in each study. The complete dataextraction table can be found in Online Resource 2 (OSM). 


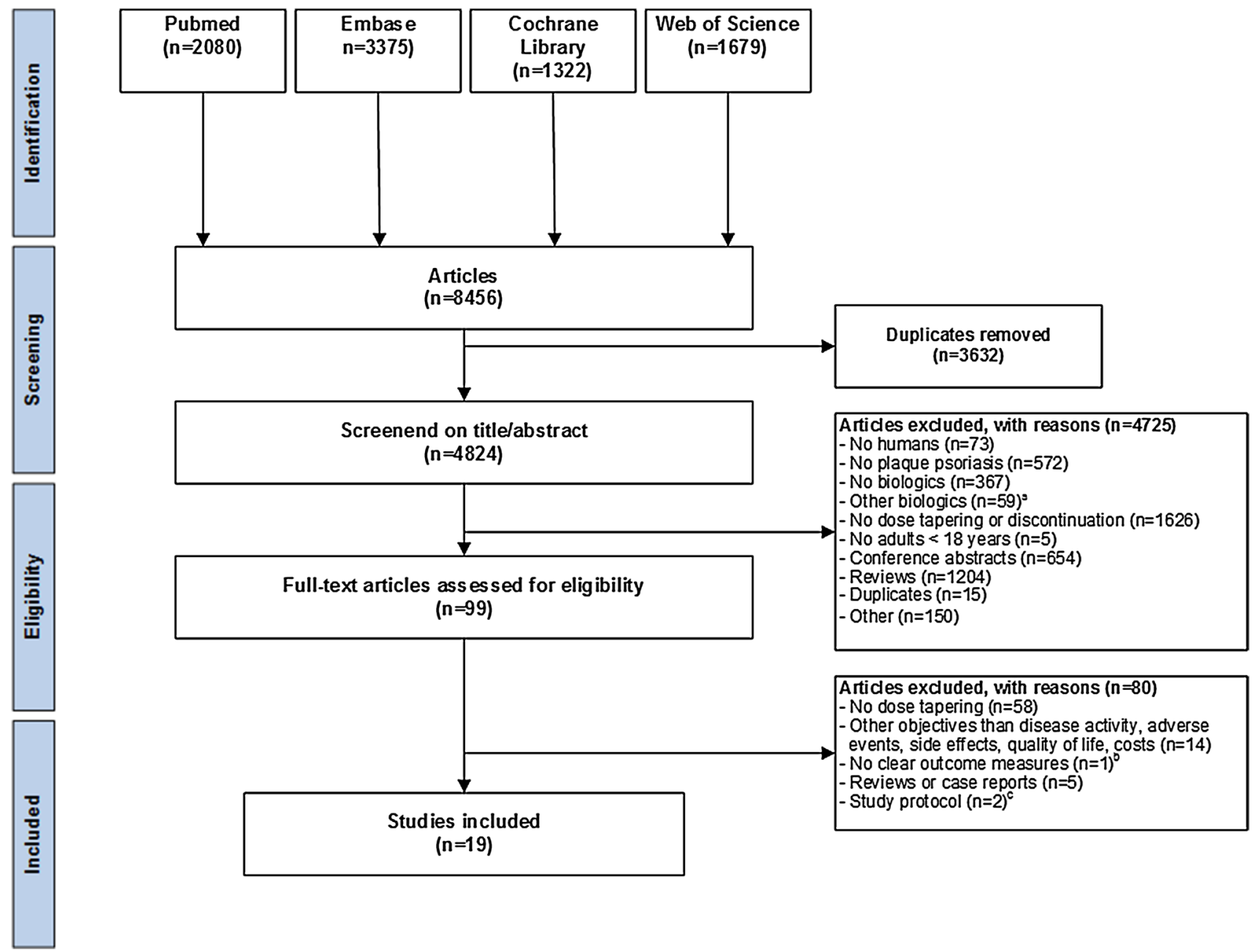

Figure 1 Flow diagram of the study selection process. ${ }^{\text {a Biologics }}$ not registered for treatment of psoriasis in Europe at the time of the review. ${ }^{\text {b}}$ The study by Na et al. [40] on dose tapering of etanercept did not specify the percentage of patients who reached PASI75 at week

\subsection{Tapering Strategy}

\subsubsection{Tapering Eligibility Criteria}

Twelve of the 19 studies proposed disease activity criteria to determine tapering eligibility $[12,13,15-17,19,20,23$, $24,26,27,29]$. Five studies did not report $[21,22,25,28$, $30]$ and two studies did not require achievement of certain disease activity criteria prior to tapering [14, 18]. Most studies used the percentage of PASI improvement compared to baseline, for example, PASI90 or PASI75, as a tool to define low disease activity. Two studies required PASI100 before starting dose tapering $[23,24]$, three studies required PASI90 [15, 17, 19], one study required either PASI90 or PASI100 [29], and one study required at least a PASI75 [26]. In contrast to relative scores, the absolute PASI was used in six studies [12, 16, 17, 20, 26, 27], of which two studies
12. Other reported outcomes did not fall within the scope of this review. This study was therefore excluded. ${ }^{\text {P Publication of protocol }}$ only

combined the absolute with a relative PASI in their criteria for tapering $[17,26]$. When PASI75 was achieved, the absolute PASI also had to be $<5$ in the study by López-Ferrer et al. [26]. In the study by Ovejero-Benito et al., patients had to achieve PASI90 or an absolute PASI $<3$ before they were considered eligible for dose tapering [17]. In the prospective study of van Bezooijen et al., stable low disease activity was defined as an absolute PASI $<8$, which was not allowed to fluctuate $\geq 3$ points during 6 weeks prior to study initiation [16]. In line with studies requiring PASI100, Bardazzi et al. and Piaserico et al. demanded an absolute PASI of 0 [20, 27]. The RCT by Atalay et al. was the only study to combine a QoL measure with an absolute PASI in their tapering eligibility criteria; an absolute PASI $\leq 5$ and a Dermatology Life Quality Index (DLQI) score of $\leq 5$ was required [12]. The Physician Global Assessment (PGA) score was used in two studies, wherein Blauvelt used the dynamic, 


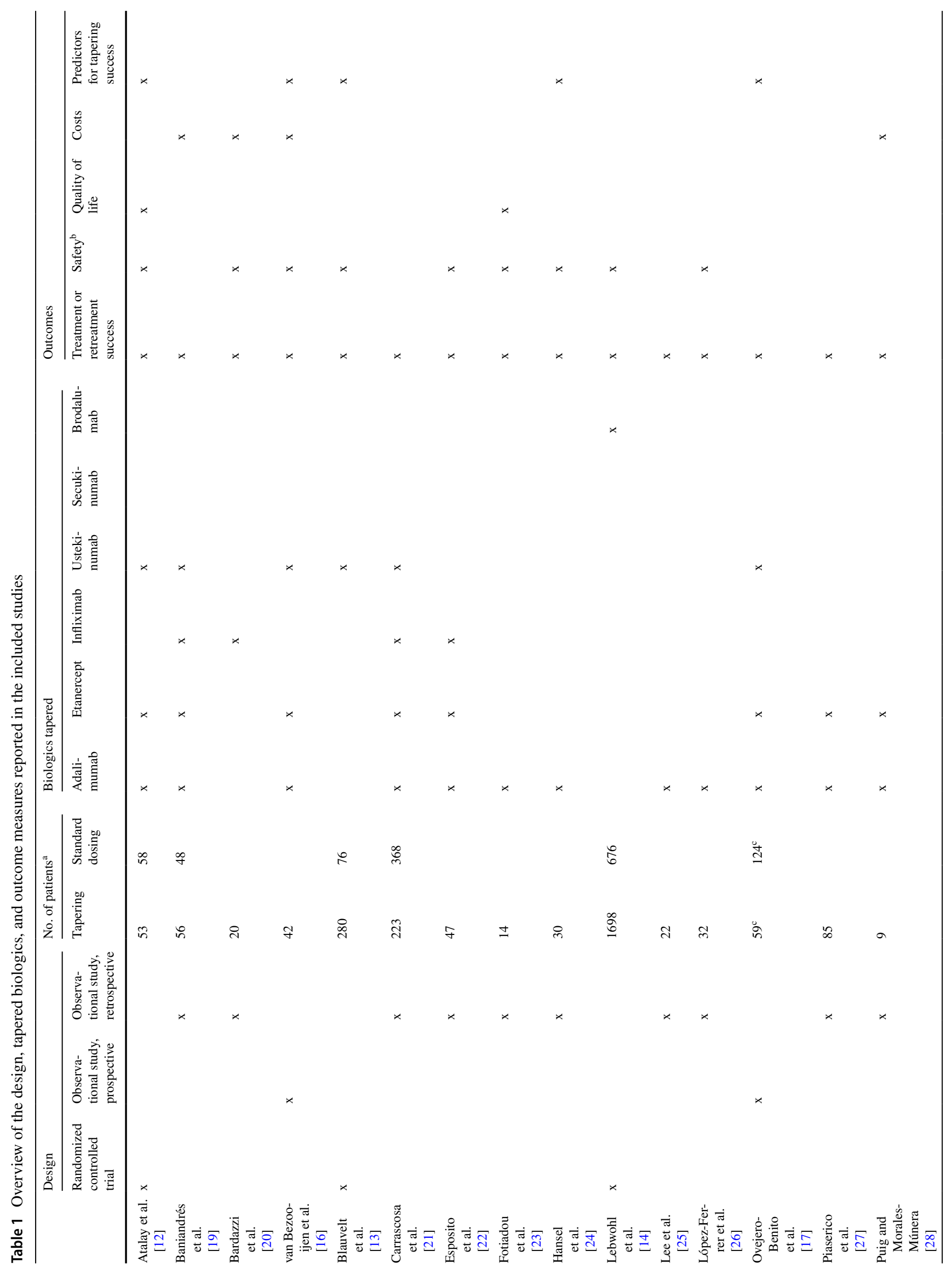




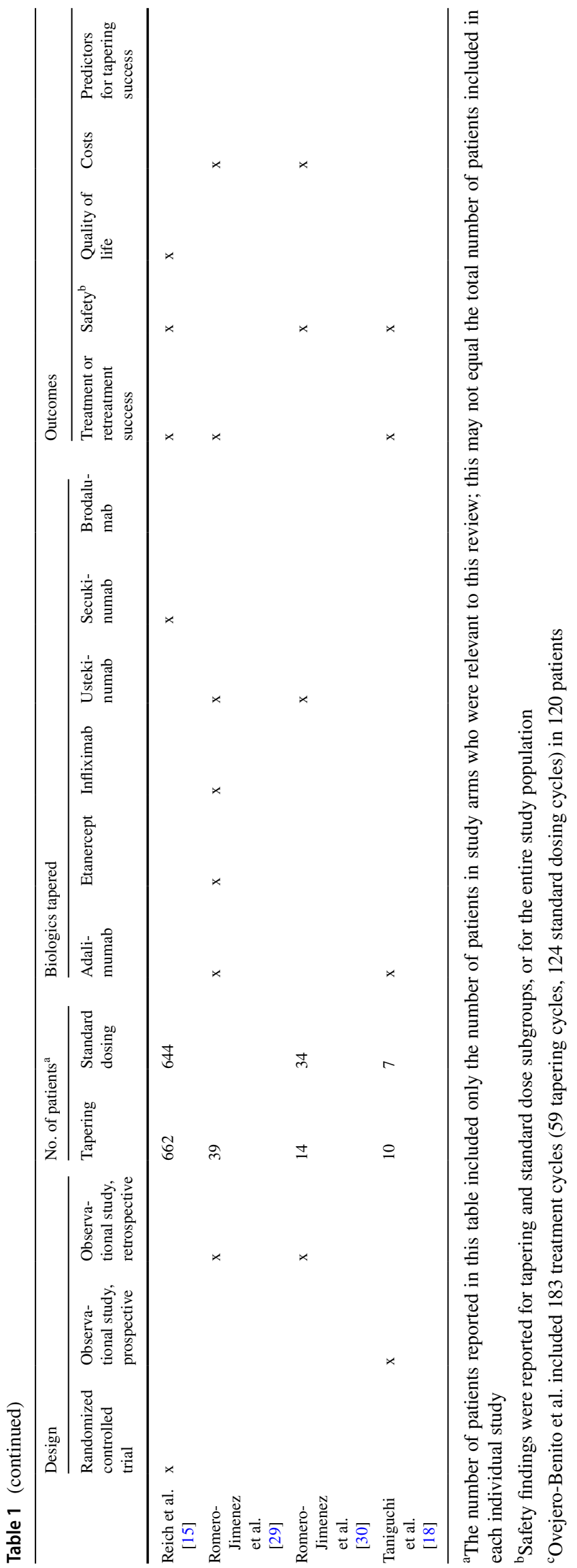

$\Delta$ Adis 


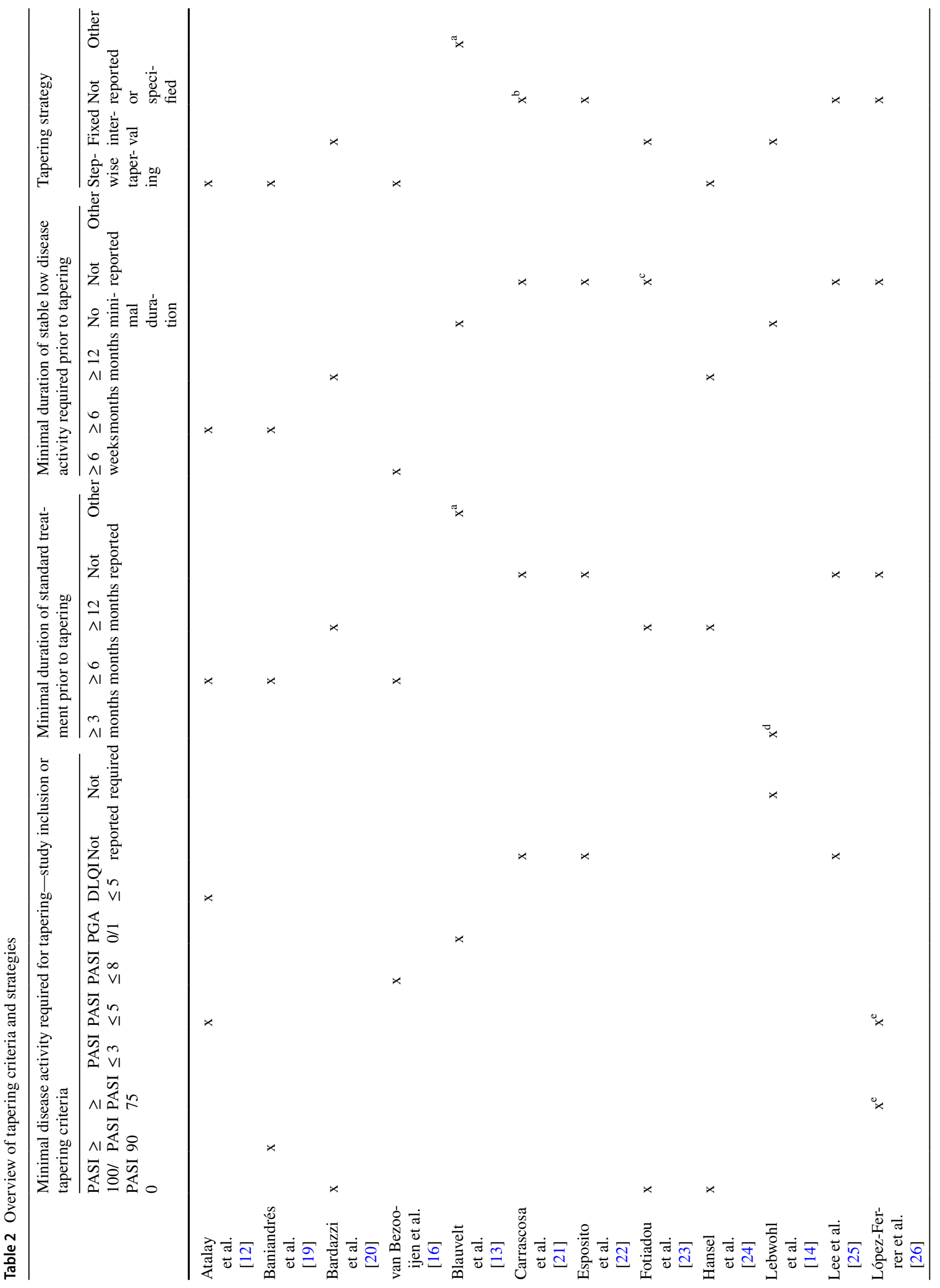




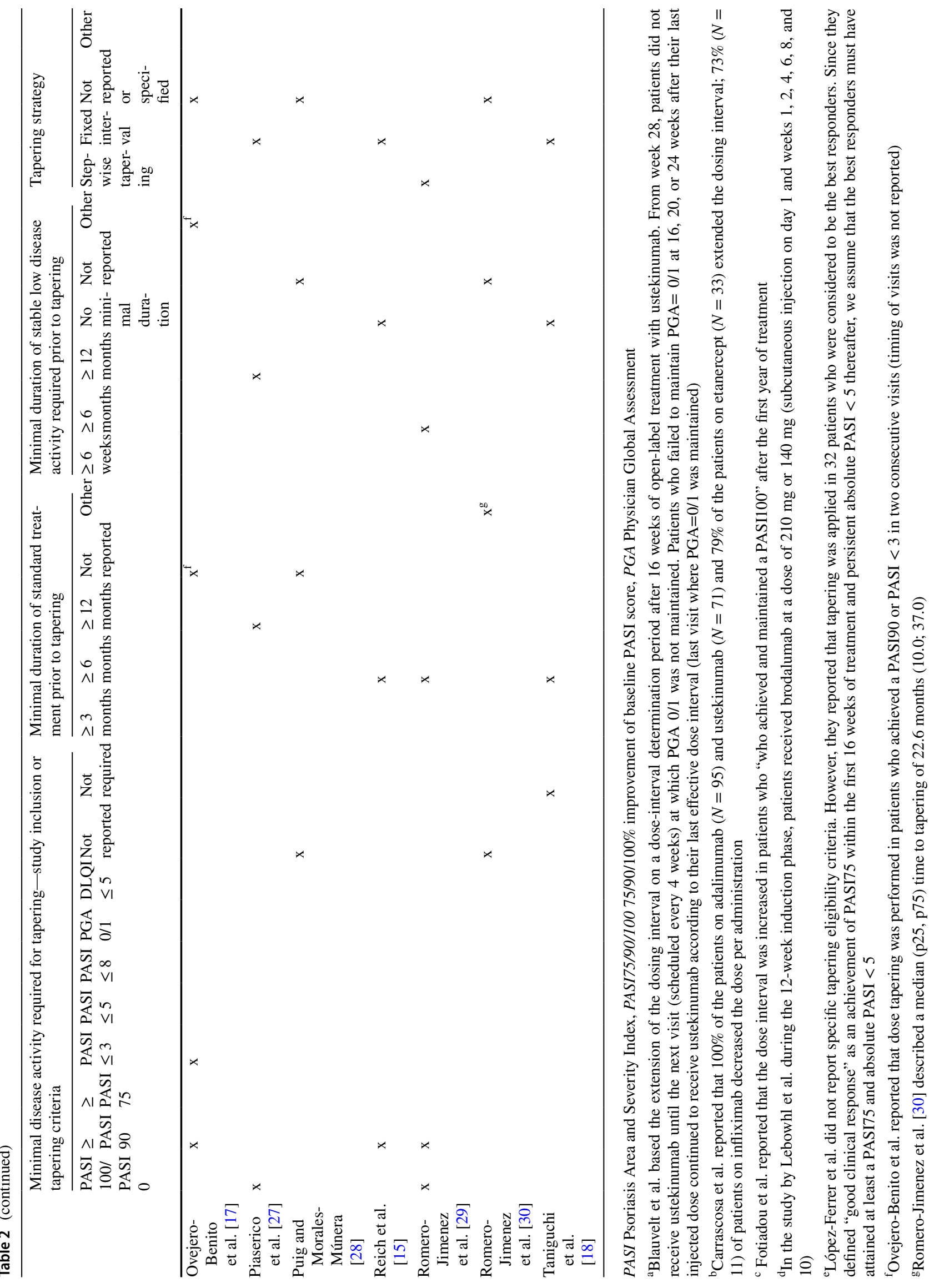


and Lebwohl used the static form of the PGA (sPGA) [13, 14]. The other studies did not mention objective criteria but selected patients based on "well-controlled disease" [25], "prolonged remission" [22], or "patient preferences" [18]. The minimal period of standard treatment required prior to dose tapering ranged from 3 months to $\geq 1$ year, with the majority of studies requiring a minimal period of 6 months (Table 1). Although definitions of disease control varied between the diverse study designs, almost all studies required a certain state of clinical remission or low disease activity before exposing patients to tapering.

\subsubsection{Tapering Regimens}

Biologic therapies can be tapered by either reducing the dose per administration or by prolonging the time interval between doses. In most studies, the biologic was initiated according to the registered standard dose, and was tapered after a certain period of time, when predefined tapering criteria of disease control were met. The prolongation of the injection intervals was performed either based on a predetermined fixed interval (single step) or performed stepwise, guided by clinical response. Only Blauvelt et al. used a different approach, in which they based the extension of the dosing interval on a dose-interval determination period after 16 weeks of open-label treatment with ustekinumab [13]. From week 16, patients did not receive ustekinumab until the next visit (scheduled every 4 weeks) at which PGA 0/1 was not maintained. Patients who failed to maintain PGA 0/1 at 16,20 , or 24 weeks after their last injected dose continued to receive ustekinumab according to their last effective dose interval (last visit were PGA 0/1 was maintained). The tapering regimens applied are summarized in Online Resource 3 (OSM).

Stepwise tapering was applied in five studies $[12,16$, 19, 24, 29]. Atalay et al. prolonged intervals between doses of adalimumab, etanercept, and ustekinumab, leading to a reduction of $33 \%$ of the original dose [12]. If patients still had a PASI or DLQI $\leq 5$ after 3 months, the interval was prolonged further, leading to $50 \%$ reduction of the original dose. Likewise, van Bezooijen et al. evaluated the effect of their dose-reduction strategy in adalimumab, etanercept, and ustekinumab every 12 weeks, and prolonged the intervals between dosages over a 42-week period [16]. This led to a minimum of $50 \%$ of the original dose for as long as the patient was satisfied, and the PASI remained $<8$. As their initial step, Romero-Jimenez et al. lengthened the interval of etanercept by 3 days ( $33 \%$ dose decrease), and the intervals of adalimumab, ustekinumab, and infliximab by 1 week [29]. If the patient was still in remission after 6 months, the interval could be further lengthened in the same manner. In patients who maintained a PASI100 on adalimumab, Hansel et al. progressively extended their dosing interval by 3-4 days each month, until a 21- or 28-day interval was reached [24]. Baniandrés et al. also performed stepwise tapering in patients who remained at PASI90-100, but their tapering protocol was not reported [19]. All other studies did not report their tapering strategy, or tapered with a fixed interval (single step). In summary, dose reduction with $33 \%$ or $50 \%$ of the original dose was most often applied and most thoroughly investigated for adalimumab, etanercept, and ustekinumab. Hereafter, we discuss the tapering regimens applied for each biologic separately.

\subsubsection{Tapering Regimens with Adalimumab}

Fourteen of the 19 included studies described dose tapering in patients on adalimumab [12, 16-19, 21-29]. In most studies, adalimumab was tapered from the standard dosing of $40 \mathrm{mg}$ Q2W to $40 \mathrm{mg}$ Q3W [12, 19, 23, 24, 26, 27, 29] or 40 mg Q4W [12, 16, 18, 19, 24-26]. Only Baniandrés et al. reported even further prolongation of the dosing interval to $40 \mathrm{mg}$ adalimumab Q6W, which had been applied in two patients [19].

\subsubsection{Tapering Regimens with Etanercept}

Etanercept was evaluated in nine studies $[12,16,17,19,21$, 22, 27-29], of which five reported their tapering regimens $[12,16,19,27,29]$. The currently registered maintenance dose of etanercept is $50 \mathrm{mg}$ once weekly $(\mathrm{QW})$, with the possibility to double the dose in the first 12 weeks of treatment. Etanercept was tapered to $50 \mathrm{mg}$ every 10 days (Q10D) [12, 19, 27, 29] or Q2W [12, 16, 19] or $25 \mathrm{mg}$ Q10D [19].

\subsubsection{Tapering Regimens with Infliximab}

Four out of five studies on infliximab reported their tapering regimens [19-22, 29]. Infliximab dosing intervals were tapered from the standard dosing of $5 \mathrm{mg} / \mathrm{kg}$ Q8W to $5 \mathrm{mg} /$ kg Q9W [19, 29], Q10W [20] or Q11W [19]. None of the included studies on infliximab evaluated a reduced dosing per administration.

\subsubsection{Tapering Regimens with Ustekinumab}

Eight studies evaluated dose tapering in ustekinumab, for which the standard maintenance dose is either $45 \mathrm{mg}$ (in patients weighing $<100 \mathrm{~kg}$ ) or $90 \mathrm{mg}$ (in patients weighing $\geq 100 \mathrm{~kg}$ ) Q12W [12, 13, 16, 17, 19, 21, 29, 30]. Four studies did not report a tapering regimen [17, 19, 21, 30]. Both relatively small interval prolongations up to $45 \mathrm{mg}$ Q13W or Q14W [29] and larger prolongations up to $45 \mathrm{mg}$ or 90 mg Q24W $[12,13,16]$ have been described. 


\subsubsection{Tapering Regimens with Interleukin-17 and Interleukin-23 Inhibitors}

Only two studies were available on dose tapering in interleukin-17 (IL-17) and interleukin (IL-23) inhibitors [14, 15]. In the study by Reich et al., the standard maintenance dose interval of secukinumab (300 $\mathrm{mg} \mathrm{Q} 4 \mathrm{~W}$ ) was extended to $300 \mathrm{mg}$ Q6W [15]. Lebwohl et al. reported on tapering of brodalumab (standard maintenance dose $210 \mathrm{mg} \mathrm{Q} 2 \mathrm{~W}$ ), and reduced the dose to $140 \mathrm{mg}$ Q2W, $140 \mathrm{mg} \mathrm{Q} 4 \mathrm{~W}$, or $140 \mathrm{mg}$ Q8W [14].

Literature on dose tapering of certolizumab pegol, ixekizumab, guselkumab, risankizumab, or tildrakizumab for psoriasis was not yet available.

\subsection{Success of Biologic Tapering}

In most cases, tapering eligibility criteria were also used as tapering success criteria. Therefore, due to the variability in tapering eligibility criteria, definitions of tapering success also varied among the included studies. Tapering outcomes were reported separately for each type of biologic, or for various types of biologics combined. For the sake of clarity, the outcomes are discussed for each biologic separately.

\subsubsection{Outcomes of Adalimumab Tapering}

Outcomes of adalimumab tapering were reported in 11 studies [12, 16-19, 23-27, 29], of which five studies focused solely on adalimumab [18, 23-26]. Four out of five observational studies that only assessed adalimumab had a retrospective approach [23-26]. Fotiadou et al. and Hansel et al. reported on tapering of adalimumab in relatively small groups (resp. $N=14$ and $N=30$ ), wherein adalimumab was tapered to $40 \mathrm{mg} \mathrm{Q3W} \mathrm{[23,} \mathrm{24]} \mathrm{and/or} \mathrm{to} \mathrm{Q4W} \mathrm{[24]} \mathrm{in}$ patients who had achieved PASI100. Fotiadou et al. started dose tapering after the first year of adalimumab treatment, and reported that $100 \%(N=14)$ maintained a PASI100 response throughout the study, with 10/14 patients having completed 30 months of adalimumab treatment at data lock [23]. In the study by Hansel et al., 30 patients started dose tapering after 1 year, and were followed-up for 4-7 years [24]. In this study, $60 \%(N=18)$ was able to maintain complete clearance throughout the study. In López-Ferrer et al. and Lee et al., the exact tapering criteria/treatment regimens were not specified [25, 26]. López-Ferrer et al. reported a treatment retention rate (drug survival) of $97 \%$ (31/32 patients) in patients on lengthened intervals, from which we deduced that these patients must have retained at least PASI50 or absolute PASI $<5$ on their tapered dose (definition of "adequate response" in this study) [26]. Lee et al. reported that in patients with remission, 8/9 (89\%) maintained "good control" for $\geq 6$ months after dose reduction
[25]. In an observational, prospective study by Taniguchi et al., 17 patients were allocated to adalimumab Q2W $(N=$ $7)$ or Q4W $(N=10)$ after 24 weeks of standard dosing [18]. At week 60, a PASI75 was achieved by nine patients $(90 \%)$ in the tapered group, and six patients $(86 \%)$ in the standard dosing group. Furthermore, PASI50 (90\% vs. 100\%) and PASI90 (40\% vs. 57\%) response at week 60 were also comparable between the tapering and standard-dosing group, respectively ( $p=1$ and $p=0.63$ ).

Tapering outcomes of adalimumab, alongside other biologics, were reported in one RCT [12] and five observational studies [16, 17, 19, 27, 29]: in the pragmatic RCT by Atalay et al., 20/23 patients (87\%) who had tapered adalimumab to $40 \mathrm{mg}$ Q3W or Q4W, were still on a low dose at the end of the study (12 months) [12]. Sixteen out of these 20 patients (70\%) had both a PASI and a DLQI $\leq 5$ at 12 months. In a prospective study by Ovejero-Benito et al., 26/27 (96\%) patients were able to maintain PASI90 or PASI $<3$ on a reduced dose for $\geq 6$ months [17]. Piaserico et al. performed a retrospective study on patients who tapered to $40 \mathrm{mg}$ Q3W at baseline [27]. Relapse was defined as loss of PASI50. The cumulative relapse risk at months 3,6 , and 12 was $0 \%$, $12 \%$, and $20 \%$ in 30 patients, respectively. The mean time to relapse was 48 months [95\% confidence interval $(95 \%$ CI) 43-52.7]. Van Bezooijen et al. performed a prospective cohort study, with a total follow-up of 78 weeks [16]. Successful dose reduction was defined as no loss of self-reported efficacy, or deterioration of the PASI $>8$. Seven out of 16 patients (44\%) were able to successfully prolong their dose interval, of whom six patients tapered to $40 \mathrm{mg}$ Q $4 \mathrm{~W}$, and one patient tapered to $40 \mathrm{mg}$ Q3W. Six patients (38\%) were unable to taper their dose interval, and three patients (19\%) were lost to follow-up. In a cross-sectional study, Baniandrés et al. reported on 30 patients who tapered adalimumab [19]. The study showed data of 19 patients on adalimumab Q3W, nine patients on adalimumab Q4W, and two patients on adalimumab Q6W. All of them maintained at least their initial PASI90 response during follow-up (mean follow-up 38 months). In a retrospective study, Romero-Jimenez et al. [29] reported on patients who tapered to $40 \mathrm{mg}$ Q3W. The dosing interval could be further lengthened if the patients remained in clinical remission for another 6 months. All of the 23 patients maintained their PASI90-100 response at time of evaluation $(<1$ year after tapering, exact duration unknown). In a retrospective study by Puig et al., tapering strategy and duration of follow-up was not reported [28]. However, they reported that six patients, who were in the maintenance phase of treatment $(>24$ weeks since start of treatment) were able to retain their PASI75 and absolute PASI $<5$ on a reduced adalimumab dose. 


\subsubsection{Outcomes of Etanercept Tapering}

Treatment outcomes of etanercept tapering were reported in one RCT [12], two prospective [16, 17], and four retrospective [19, 27-29] observational studies. In the patients on etanercept from the RCT by Atalay et al., 6/13 patients (46\%) who had tapered etanercept to $50 \mathrm{mg}$ Q10D or Q2W, were still on a tapered dose at the end of the study (12 months) [12]. Three patients (23\%) had both an absolute PASI and DLQI $\leq 5$ after 12 months. Baniandrés et al. reported that in their cross-sectional study, two patients on etanercept $25 \mathrm{mg}$ Q10D, one patient on etanercept 25 $\mathrm{mg} \mathrm{QW}$, five patients on etanercept $50 \mathrm{mg}$ Q10D, and two patients on etanercept $50 \mathrm{mg}$ Q2W were included [19]. All of them maintained a $\geq$ PASI90 response during followup (mean follow-up 38 months). In another retrospective study, Romero-Jimenez et al. [29] reported nine patients who tapered to $50 \mathrm{mg}$ Q10D. All of them maintained their PASI90-100 response at the time of evaluation $(<1$ year after tapering). In the prospective study by Ovejero-Benito et al., all three included patients (100\%) were able to maintain this response on a reduced dose $\geq 6$ months (tapering strategy/regimen not reported) [17]. In the retrospective study of Piaserico et al. the dose interval was increased to $50 \mathrm{mg}$ Q10D in 54 patients at baseline [27]. The cumulative relapse (loss of PASI50) risk at month 3,6 , and 12 was respectively $14 \%, 31 \%$, and $39 \%$. In the prospective cohort study of van Bezooijen et al., 5/16 patients (31\%) were able to successfully prolong their dose interval to 50 mg Q2W (no loss of self-reported efficacy, or deterioration of the PASI > 8) [16]. Nine patients $(56 \%)$ were unable to taper their dosing interval. In the retrospective study of Puig et al., it was reported that three patients on a tapered dose of etanercept maintained their PASI75 and absolute PASI $<5$ during the entire study (strategy and follow-up not reported) [28].

\subsubsection{Outcomes of Infliximab Tapering}

Treatment outcomes of infliximab tapering were reported in three retrospective studies [19, 20, 29]. Romero-Jimenez et al. [29] reported on two patients who had tapered infliximab from $5 \mathrm{mg} / \mathrm{kg}$ Q8W (standard dosing) to $5 \mathrm{mg} / \mathrm{kg}$ Q9W [29]. Both patients retained their initial PASI90-100 response at evaluation ( $<1$ year after tapering). Bardazzi et al. retrospectively analyzed 20 patients who had reached PASI 0 and prolonged their dosing interval to $5 \mathrm{mg} / \mathrm{kg}$ Q10W [20]. Disease-activity outcomes or follow-up period were not reported, but relapses occurred in five patients (25\%). Baniandrés et al. tapered infliximab in two patients to $5 \mathrm{mg} / \mathrm{kg}$ Q9W, and one patient to $5 \mathrm{mg} / \mathrm{kg}$ Q11W [19]. All of them maintained at least their PASI90 response during follow-up (mean follow-up 38 months).

\subsubsection{Outcomes of Ustekinumab Tapering}

Treatment outcomes of ustekinumab tapering were discussed in two RCTs [12,13], two prospective [16, 17], and two retrospective studies $[19,29]$. In a randomized phase IIIb trial by Blauvelt et al., dosing intervals were prolonged to once every 16, 20, or 24 weeks [13]. In patients who administered ustekinumab Q16W, Q20W, or Q24W, PASI75 was maintained until week 112 in, respectively, $65.6 \%(N=40)$, $60.8 \%(N=31)$, or $82.1 \%(N=69)$, compared to $69.7 \%(N=$ $53)$ in the standard dosing group. In this study, only $30 \%(N$ $=84$ ) of the patients who achieved PGA 0/1 at week 28 were not able to prolong their dosing interval up to Q16W. In the RCT by Atalay et al., 10/17 patients (59\%) in the tapering group (administration of ustekinumab $45 \mathrm{mg} / 90 \mathrm{mg}$ Q18W or Q24W) were still on a lower dose after 12 months [12]. At this timepoint, nine patients (53\%) had both an absolute PASI and DLQI $\leq 5$. In the prospective study by OvejeroBenito et al., 22/29 patients (76\%) were able to maintain their initial PASI90 or PASI $<3$ response on a reduced dose for $\geq 6$ months [17]. Van Bezooijen et al. included nine patients on standard dosing of $45 \mathrm{mg}$ ustekinumab Q12W, and one patient on $90 \mathrm{mg}$ Q12W in their prospective cohort study [16]. At week 78, in the patients who received the 45 mg dosing, 2/9 patients (22\%) had been able to successfully prolong their dose interval to $45 \mathrm{mg}$ Q24W (no loss of self-reported efficacy, or deterioration of the PASI $>8$ ). Seven patients $(77 \%)$ failed to taper their dosing interval. The single patient on a per-label dose of $90 \mathrm{mg}$ Q12W also failed to taper. In a retrospective study, Baniandrés et al. tapered ustekinumab to $45 \mathrm{mg}$ Q13W in 11 patients (85\%) and to $45 \mathrm{mg}$ Q14W in two patients (15\%) [19]. All of them maintained $\geq$ PASI90 response during follow-up (mean follow-up 38 months). Romero-Jimenez et al. reported that five patients were able to maintain their PASI90-100 response on a tapered dose of $45 \mathrm{mg}$ ustekinumab Q13W at evaluation ( $<1$ year after tapering) [29].

\subsubsection{Outcomes of Secukinumab Tapering}

The article by Reich et al. was the only one to explore tapering of secukinumab [15]. In this randomized open-label study, 662 patients on secukinumab tapered their dose to 300 mg Q6W, after having received the standard dose $(300 \mathrm{mg}$ $\mathrm{Q} 4 \mathrm{~W})$ for 24 weeks. The standard dosing group included 644 patients. PASI90 response was maintained at week 52 in $85.7 \%$ of patients in the standard dosing group versus $74.9 \%$ in the tapered group [odds ratio (OR) $1.91,95 \%$ CI 1.44-2.55]. Neither strategy differed markedly for PASI50 (99.2\% in the tapering group vs. $99.7 \%$ in the maintenance group) and PASI75 (93.5\% vs. $97.9 \%$ ) responses at week 52. 


\subsubsection{Outcomes of Brodalumab Tapering}

The study by Lebwohl et al. was the only one to report on brodalumab tapering [14]. They combined the results of two randomized phase III studies (AMAGINE-2 and 3), in which, amongst other treatments, brodalumab maintenance treatment (210 mg Q2W) was compared to tapered doses. In both studies, after 12 weeks of brodalumab treatment with either $210 \mathrm{mg}$ or $140 \mathrm{mg}$ on a normal induction scheme, patients were rerandomized to standard dosing, or allocated to one of the tapering subgroups, receiving $140 \mathrm{mg}$ brodalumab Q2W, Q4W or Q8W. In the patients on $140 \mathrm{mg}$ brodalumab Q2W ( $N=337$ AMAGINE-2, $N=343$ AMAGINE-3), approximately $44 \%(N=144, N=154)$ was able to maintain their sPGA score of $0 / 1$ at week 52 , compared to $62 \%(N$ $=209, N=208)$ of all patients using the standard dose $(N$ $=334, N=342)$. In subgroups receiving $140 \mathrm{mg}$ Q4W $(N$ $=335, N=341)$ or $\mathrm{Q} 8 \mathrm{~W}(N=168, N=174)$, maintaining SPGA $0 / 1$ response was possible in $12.3 \%$ and $5.3 \%$ of patients, respectively. A significant difference in maintaining a sPGA score of 0/1 was reported between the standard dose and all tapering subgroups. This significant difference was also found when the $140 \mathrm{mg}$ Q2W group was compared to the $140 \mathrm{mg}$ Q4W or Q8W subgroups $(p<0.001)$.

\subsubsection{Studies Reporting Tapering Outcomes for Various Biologics Combined}

Two studies did not report tapering outcomes separately for each biologic studied, but clustered all patients who had been able to taper any sort of biologic into one group [21, 22]. In a cross-sectional study on a subset of patients from the BIOBADADERM registry, tapering of multiple biologics (adalimumab, etanercept, ustekinumab, and infliximab) was reported in 223 patients [21]. The majority of tapered patients used adalimumab $(N=95,42.6 \%)$. At time of evaluation, a mean PASI score of 1 was reported for the patients in the tapered group ( $N=140$, data not available for entire group), compared to a mean PASI of 2.6 in patients on the standard dose $(N=231)$. In the retrospective study of Esposito et al., 50 patients on adalimumab, etanercept or infliximab tapered their dose (tapering strategy not specified) [22]. At week 96, the PASI75 response rate was higher in patients on a tapered dose $(N=50)$ compared to patients on standard maintenance treatment $(N=280)$, although the difference between both groups was not significant (details not reported). In this study, patients with a temporary treatment interruption were also included in the dose-reduction group.

In the pragmatic, RCT by Atalay et al., dose tapering was compared to usual care in patients on adalimumab, etanercept, or ustekinumab [12]. Outcomes for each biologic separately have been discussed in the paragraphs above. For the total group, 28 (53\%) of the 53 tapered patients, tapering was deemed successful (low dose and low DLQI/PASI) at 12 months. This study reported that median PASI after 12 months was 3.4 for the tapering group, and 2.1 for the group on standard dosing (non-inferiority for dose tapering was not demonstrated based on PASI). This was the only study that made a distinction between short (single PASI and/or DLQI $\geq 5$ ) and persistent (PASI and/or DLQI $\geq 5$ during $\geq 3$ months) flares. Short flare-ups were reported in 19 patients $(36 \%)$ in the tapering group and eight patients $(14 \%)$ in the group on standard dosing $(p=0.04)$. Persistent flare-ups were reported in $9 \%$ (five and five patients, respectively) for both groups $(p>0.99)$.

\subsection{Retreatment After Relapse}

Retreatment strategies were reported in nine studies [12, 14, $16,19,20,24,26,27,29]$. The criteria used to define loss of clinical efficacy, as a condition for retreatment, were highly variable among studies. Criteria that were used are: absolute PASI $>5$ and/or DLQI $>5$ [12], unacceptable increase in disease activity according to the patient and/or PASI $>8$ [16], loss of PASI90-100 response [19, 29], loss of PASI50 response [20], loss of 50\% PASI improvement [27], PGA $\geq 3$ or persistent sPGA scores of 2 during $\geq 4$-weeks [14], or simply "loss of response" (undefined) [26]. Hansel et al. defined relapse as a loss of $\geq 20 \%$ PASI improvement [24]. Since they only included patients with a PASI100, this definition of relapse corresponds to a loss of PASI80. In case of a relapse, patients returned to their previous most effective dose $[12,16,26,29]$ or standard regimen $[14,19,20$, $24,27]$. Effectiveness after retreatment was reported in two retrospective studies [24, 27] and one RCT [12]. Hansel et al. reported that 12 patients who were unable to maintain PASI100 on a tapered adalimumab dose, regained PASI100 within 1 month after retreatment with standard dosing [24]. Likewise, all patients in the study by Piaserico et al. on a tapered dose of etanercept $(N=21)$ or adalimumab $(N=$ 6) who experienced a relapse showed a rapid response [27]. Atalay et al. reported that in the dose-reduction group, the first persistent flare (PASI and/or DLQI $\geq 5$ during $\geq 3$ months) occurred after 7.5 months [12]. Four out of five patients in the dose-reduction group who developed persistent flares thereafter had not returned to a PASI $\leq 5$ at evaluation at 12 months. It is not stated if these patients regained their response after a longer period of time.

\subsection{Safety}

The frequency of adverse events (AEs) or serious adverse events (SAEs) was reported in 12 studies [12-16, 18, 20, 22-24, 26, 30]. Six studies reported AEs and/or SAEs separately for patients receiving usual care and for patients on a tapered dose $[12-16,18]$. In these studies, similar 
proportions of patients with AEs and/or SAEs were reported in both groups, with a maximum follow-up of 96 weeks after the initiation of dose tapering [13]. Except for a higher rate of general non-specific musculoskeletal complaints in patients on reduced doses of adalimumab, etanercept, or ustekinumab in the study by Atalay et al. (rate ratio 4.92; 95\% CI 2.04-11.87; $p<0.001$ ), no significant differences in AEs were reported between the two groups [12]. The article by Blauvelt et al. was the only article that reported anti-drug antibody (ADA) development in their safety analysis, in patients on ustekinumab who extended their dosing intervals up to 24 weeks [13]. In this study, the incidence of ADA development was similar among patients in the tapered group $(N=32,11 \%)$ and the maintenance group $(N=7$, $9 \%$ ). The studies included in this scoping review did not report any safety issues assumed to be causally related to dose tapering.

\subsection{Predictors for Successful Dose Tapering in Psoriasis}

To date, the available evidence on predictors for successful tapering is limited. In a prospective cohort of 120 patients (183 cycles of treatment with adalimumab, etanercept, or ustekinumab), Ovejero-Benito et al. reported that polymorphism rs1008953 (AG in SDC4) was associated with successful dose reduction (PASI90 or PASI $<3$ over $>6$ months) in patients on adalimumab (OR 204.11; 95\% CI $2.67-1.6 \times 10^{4}, p=0.016$ ), whilst polymorphisms rs4649203 (AG/GG inIL28RA) (OR 0.21; 95\% CI 0.06-0.72, $p=0.025)$, rs 11096957 (CC in TLR10) (OR 0.00; 95\% CI $0.00-0.15, p=0.011), \mathrm{rs} 240993$ (CT/TT in TRAF3IP2) (OR 0.02 ; $95 \%$ CI $0.00-0.23, p=0.002$ ) and rs6934187 (CC/ CG in MICA-A9) (OR 0.02; 95\% CI 0.00-0.49, $p=0.040$ ) were associated with failure of tapering [17]. Furthermore, a significant positive association between successful dose reduction in adalimumab and male sex was found (authors' correspondence). In patients on ustekinumab, polymorphism rs983332 (AC in LMO4) (OR 13.12; 95\% CI 1.91-89.98, $p=0.009)$ and rs10494292 (GT/GG in LELP1) (OR 9.28; 95\% CI 1.47-58.37, $p=0.018$ ) were positively associated with successful tapering, whereas rs 10754555 (CG/GG in $N L R P 3$ ) (OR 0.11; 95\% CI 0.02-0.68, $p=0.017$ ) showed a negative association. In patients on adalimumab, etanercept, and ustekinumab, van Bezooijen et al. evaluated the predictive value of the baseline serum trough concentrations, which were found to be unpredictive for successful doseinterval prolongation [16]. Various clinical parameters have been explored with regard to successful dose reduction. In an RCT, Atalay et al. looked for clinical predictors for successful dose tapering but no variables showed a predictive value [12].
As opposed to predictors for successful tapering, Piaserico et al. reported predictors for relapse after dose reduction [27]. In a retrospective cohort study on 85 patients, they reported that age $<52$ years [hazard ratio (HR) 2.6, $p$ $=0.04]$, disease duration $>20$ years (HR 3.7, $p=0.007)$, a PASI score $>15$ before tumor necrosis factor inhibitor (TNFi) treatment (HR 3.4, $p=0.01)$ and baseline C-reactive protein $(\mathrm{CRP})>1 \mathrm{mg} / \mathrm{L}$ (HR 3.7, $p=0.006$ ) were associated with relapse after dose reduction in patients on etanercept. In another retrospective cohort study of 30 patients on adalimumab by Hansel et al., a higher body mass index (BMI) turned out to be an independent predictor of relapse after dose tapering (HR 1.231, 95\% CI 1.008-1.504, $p<$ $0.05)$ [24]. In this study, patients who were successful in dose tapering also had a significantly shorter time to achieve PASI100 after initial start of adalimumab than patients who failed. Similar hypotheses were posed in three other studies $[13,15,30]$. Of note, none of these studies corrected this finding for potential confounders.

In overview, studies with high-level evidence on possible predictors for successful dose tapering were sparse, and consensus on a potential predictor for successful tapering was not reached.

\subsection{Quality of Life}

Quality of life (QOL) measurements were reported in three studies, all using the DLQI $[12,15,23]$. The study by Atalay et al. was the only study to incorporate the DLQI in their tapering criteria and strategy [12]. After 12 months, a median DLQI score of 1.0 [interquartile range (IQR) 0.0-2.0] in the tapering group, versus 0.0 (IQR 0.0-2.0) in the standard dosing group was found. The mean difference at month 12 corrected for baseline was 0.8 (95\% CI 0.3-1.3). As the non-inferiority margin in this study for DLQI was set at 2.0, this indicated that tapering was non-inferior compared to usual care, as for QOL. Reich et al. reported a small but significant difference in overall change in DLQI between the standard dosing and tapering groups [15]. The difference in change of DLQI after one year of secukinumab treatment was -0.62 (95\% CI 0.93-0.31, $p=0.0001)$, indicating a larger decrease of DLQI in the maintenance group. Fotiadou et al. reported that ten patients who continued adalimumab for 30 months, and had been tapered to $40 \mathrm{mg} \mathrm{Q} 3 \mathrm{~W}$ after 1 year of standard treatment, all reported a DLQI of 0 [23].

\subsection{Costs}

Cost savings as a result of tapering were described in six studies [16, 19, 20, 28-30]. In a retrospective cohort of 112 patients on adalimumab, etanercept, ustekinumab, and infliximab, of whom $50 \%$ received a reduced dose and $7.1 \%$ received an escalated dose, Baniandrés et al. reported 13\% 
savings for the entire cohort, corrected for the patients on an escalated dose [19]. Bardazzi et al. reported a total savings of $19.8 \%$ per year in a cohort of 20 patients who tapered infliximab [20]. Romero-Jimenez et al. reported yearly savings of $€ 115,969$ in a cohort of 118 patient on adalimumab, etanercept, ustekinumab, and infliximab, of whom 33\% received a reduced dose [29]. Romero-Jimenez et al. studied a cohort of patients on ustekinumab with various dosing regimens. Overall, these patients received on average 93.3\% of the standard dose [30]. This led to annual cost savings of $€ 771$ per patient. Van Bezooijen et al. estimated that dose tapering could result in treatment cost savings of $€ 7021$ per adalimumab-tapered patient, $€ 6939$ per etanercept-tapered patient, and $€ 7130$ per ustekinumab-tapered patient annually [16].

\section{Discussion}

In this scoping review, we summarized the available literature on dose tapering of biologics in adult patients with plaque psoriasis. We assessed tapering eligibility criteria, tapering regimens, and tapering outcomes, and included safety, QOL, predictors for successful tapering, and costs. Nineteen studies were included, for which the designs differed vastly. Treatment outcomes for dose tapering were reported in 17 studies, but only eight studies were specifically set up to evaluate the effect of dose tapering $[12,13$, $15,16,18,20,24,27]$. Since not all studies were designed to evaluate dose tapering, reporting of important components in dose tapering strategies was often incomplete. The following gaps in literature were identified: lack of uniform tapering criteria and strategies, and no long-term data on the impact of tapering on disease activity, safety, and QOL. Furthermore, finding predictors for successful tapering, and insight in tapering of the newest generation of biologics is an important topic of interest. Despite a wide variety in dosetapering strategies, based on the current evidence, biologic dose tapering seems feasible and achievable in a substantial number of psoriasis patients with (stable) low disease activity or clinical remission. Safety did not seem to be positively or negatively affected by dose-tapering strategies. In one RCT, based on the DLQI, tapering strategies were found to be non-inferior to standard maintenance dosing [12]. The effect of dose tapering appears promising with regard to substantial cost savings.

Tapering success rates differed between studies; however, all studies showed that a substantial part of patients tapered successfully. Identifying the most optimal tapering strategy based on the current literature is challenging due to the heterogeneity of tapering strategies between studies, and design differences. A solution would be to reach consensus on treatment goals. Such goals could be used to select patients (reaching this predefined goal as an eligibility criterion for tapering), to guide the tapering steps, and to define if tapering is successful or not (whether the predefined goal is maintained). In psoriasis treatment, there are various tools to measure disease severity, which can serve as a treatment goal. These include relative or absolute PASI, or a dynamic PGA or static PGA. The majority of the included studies used a relative PASI (PASI90) as an eligibility criterion for tapering, but a combination of absolute and relative PASI scores was also applied. A relative PASI can cause misclassification of disease status, since patients can still have significant disease activity after achieving PASI75 or PASI90 relative to their initial disease status. In addition, in case of a low baseline PASI, a large relative improvement is hard to achieve, and not always representative for the actual disease severity. In contrast, an absolute PASI is not dependent on baseline status, and better represents the individual disease status and its severity $[31,32]$. However, to date it has been used less often as a treatment goal in clinical studies. So far, patient-reported outcomes such as DLQI have not been integrated much in dose-tapering strategies, with only one study including a combination of an absolute PASI and DLQI in their tapering criteria [12]. Two studies included patients' preferences in their tapering strategies $[16,18]$. To pursue individualized care, it is advised to integrate QOL measurements or patients' personal treatment goals in dose-tapering strategies, as these may be more relevant to the patient than small changes in PASI. In our opinion, an absolute PASI or disease activity cut-off score offers objectivity, and is comprehensive enough for clinicians to use in daily clinical practice. When combined with the clinicians' and patients' perspective (shared decision-making), this could serve as the optimal tapering criterion.

Once stable low disease activity or clinical remission is established, tapering strategies can be discussed with the patient. Reviewing the literature, it is unclear what minimal treatment duration or duration of stable low disease activity suffices to assess tapering eligibility. Most studies required a minimal treatment period or stable low disease activity/remission of 6-12 months. Since for most biologics the length of time needed to become effective is between 6 weeks and 6 months, we estimate that a minimal period of stable low disease activity of $\geq 6$ months would be a safe option.

Regarding the dose-tapering regimen, prolonging the dose interval between administrations was a more frequently applied strategy than reducing the dose per administration. Most studies tapered through a fixed interval (single step) as opposed to using stepwise tapering, ultimately leading to a dose reduction of $33 \%$ or $50 \%$ of the original dose. Dose tapering of the biologics adalimumab, etanercept, and ustekinumab have been thoroughly investigated. (Short) Exacerbations are inherent to dose tapering as the dose-response 
balance is being challenged, and aiming to avoid periods of short-lived relapse therefore seems unrealistic. However, long-lived, persistent flares should be minimized as much as possible, for instance by lowering the dose in small steps. Also, taking large steps at once could amplify fear of relapse. Fear of relapse, in both patients and clinicians, could impede the implementation of dose-tapering strategies. In a study on the attitude of RA patients towards dose tapering, fear of relapse was reported to be one of the main concerns [33]. However, $74 \%$ of the 192 RA patients who completed the survey had a (very) positive attitude towards dose tapering in the presence of low disease activity. It would be interesting to investigate whether psoriasis patients share this opinion. In patients with RA, dose-tapering and discontinuation strategies have already been implemented in the European recommendations and are part of daily practice [8]. It has been described that the majority of RA and PsA patients who lose stable low-disease activity or clinical remission due to dose reduction recapture their previous response upon reintroduction $[34,35]$. In the present review, we found that in case of a flare, further deterioration of disease activity can be prevented by (temporarily) reinstalling the previous effective treatment interval, thereby minimizing the risk of long-lived flare ups and loss of treatment response [12, 24, 27]. However, the evidence on this issue was limited, which complicates drawing well-founded conclusions.

Dose tapering did not result in a significant increase or decrease of causally related (S)AEs, although the evidence on safety issues was limited. Theoretically, dose tapering could lead to a decrease in long-term adverse events, but long-term follow-up has not frequently been covered in the current body of evidence. ADA levels were analyzed in only one study, which showed similar proportions of ADAs in patients on a tapered or standard dose of ustekinumab [13]. A change in ADA levels in patients on adalimumab or infliximab, both notorious for potential ADA formation [36], has not been evaluated in patients on a tapered dose. Since increased ADA levels might lead to loss of response, immunogenicity of these biologics is important to consider [37]. In patients treated with infliximab, a higher incidence of serious infusion-related reactions has been reported in patients receiving intermittent doses with long intervals compared to patients on maintenance therapy [38]. Even though the studies with patients on a prolonged dose of infliximab included in this review did not raise any safety concerns, we do not advise dose tapering by interval prolongation in patients on infliximab due to the above-mentioned concerns. To date, such SAEs causally related to dose tapering in patients on adalimumab have not been described.

Thus far, studies with high-level evidence on possible predictors for successful dose tapering are lacking, and consensus on a potential predictor for successful tapering has not been reached. The studies that reported on costs provided rough estimations of the potential cost savings as a result of dose tapering, but did not provide cost-effectiveness analyses, or took indirect costs into account. However, as in general biologics are expensive, and substantial numbers of patients could taper their dose by $33 \%$ or $50 \%$, costs savings quickly become significant. The exact cost savings will greatly depend on the tapering strategy applied, the actual purchase cost of the type of biologic, and the availability of biosimilars. These factors vary in time and between countries.

A limitation of our scoping review is that title and abstract screening was done by a single reviewer, which may cause bias in the selection of articles. However, the Cochrane Handbook reports that one reviewer suffices for the title/abstract screening process, as long as the full-text screening process is executed by at least two reviewers, independently [39]. Another limitation is that the study by Lebwohl et al. was included in this review, although it did not completely fulfill the inclusion criteria [14]. Some patients in this study had received a lower dose (140 mg per administration) during the 12-week induction phase. However, as they received a tapered dose over 40 weeks and it was the only study on brodalumab tapering, we considered this study to be of added value. Inherent to the methodology of a scoping review, risk of bias and critical appraisal of the literature were not assessed [11].

In conclusion, based on this scoping review on dose tapering of biologics in adult patients with plaque psoriasis, dose tapering seems to be feasible and achievable. However, the optimal dose-tapering strategy still needs to be determined. The eligibility criteria, tapering regimens, and definitions of successful tapering differed between the included studies, influencing outcomes and reducing our ability to draw general conclusions. In addition, studies did not always report on important components of the dose-tapering strategies used, or outcomes or safety. So far, the effectiveness and safety outcomes of dose tapering are reassuring. We deliberately included studies of varying designs in order to provide more insight in this relatively unexplored field. Future research should ideally be designed as a RCT, in which usual care is preferably included as a study arm. By including a usual-care arm, it is possible to estimate if tapering leads to cost-effective and safer treatments. Moreover, there are several gaps that need to be explored, such as the optimal criteria for dose tapering, the long-term impact of tapering on disease activity, QoL, and safety, and implementation of tapering. Also, with the recent introduction of interleukin-17 and interleukin-23 inhibitors, it is important to study dosetapering possibilities in these drugs. Based on the current evidence, dose tapering has potential and can have important implications for patients and society. 


\section{Declarations}

Funding None.

Conflict of interest C. A. J. Michielsens has nothing to disclose. M. E. van Muijen carries out clinical trials for Abbvie, Celgene, Janssen, and Novartis, and has received a speaking fee from Janssen. All funding is not personal but goes to the independent Research Fund of the Department of Dermatology of the Radboud University Medical Center, Nijmegen (Radboudumc), the Netherlands. L. M. Verhoef has nothing to disclose. J. M. P.A. van den Reek has carried out clinical trials for AbbVie, Celgene, and Janssen, and has received speaking fees from/ attended advisory boards for AbbVie, Janssen, BMS, and Eli Lilly, and reimbursement for attending a symposium from Janssen, Pfizer, Celgene, and AbbVie. All funding is not personal but goes to the independent research fund of the Department of Dermatology of Radboud University Medical Center, Nijmegen, the Netherlands. E. M. G. J. de Jong has received research grants for the independent research fund of the Department of Dermatology of the Radboud University Medical Center, Nijmegen, the Netherlands, from AbbVie, Novartis, Janssen Pharmaceutica, and Leo Pharma. Has acted as a consultant and/or paid speaker for and/or participated in research sponsored by companies that manufacture drugs used for the treatment of psoriasis including AbbVie, Janssen Pharmaceutica, Novartis, Lily, Celgene, Leo Pharma, $\mathrm{UCB}$, and Almirall. All funding is not personal but goes to the independent research fund of the Department of Dermatology of Radboud University Medical Center Nijmegen, the Netherlands.

Ethics approval Not applicable.

Consent to participate Not applicable.

Consent for publication Not applicable.

Availability of data and material Data sharing is not applicable, since no datasets were generated or analyzed for the generation of the current article.

Code availability Not applicable - this article is based on previously performed studies and does not include any new studies on humans or animals conducted by any of the authors.

Author contributions All authors meet the International Committee of Medical Journal Editors (ICMJE) criteria for authorship of this article and take responsibility for the integrity of the work. All authors read and approved the publication of the final manuscript.

Open Access This article is licensed under a Creative Commons Attribution-NonCommercial 4.0 International License, which permits any non-commercial use, sharing, adaptation, distribution and reproduction in any medium or format, as long as you give appropriate credit to the original author(s) and the source, provide a link to the Creative Commons licence, and indicate if changes were made. The images or other third party material in this article are included in the article's Creative Commons licence, unless indicated otherwise in a credit line to the material. If material is not included in the article's Creative Commons licence and your intended use is not permitted by statutory regulation or exceeds the permitted use, you will need to obtain permission directly from the copyright holder. To view a copy of this licence, visit http://creativecommons.org/licenses/by-nc/4.0/.

\section{References}

1. Menter A, Gottlieb A, Feldman SR, Van Voorhees AS, Leonardi $\mathrm{CL}$, Gordon KB, et al. Guidelines of care for the management of psoriasis and psoriatic arthritis: section 1. Overview of psoriasis and guidelines of care for the treatment of psoriasis with biologics. J Am Acad Dermatol. 2008;58(5):826-50. https://doi. org/10.1016/j.jaad.2008.02.039.

2. Zorginstituut Nederland. https://www.medicijnkosten.nl/. Accessed 24 Sep 2020.

3. Thomaidou E, Ramot Y. Injection site reactions with the use of biological agents. Dermatol Ther. 2019;32(2):e12817. https://doi. org/10.1111/dth.12817.

4. Scherer K, Spoerl D, Bircher AJ. Adverse drug reactions to biologics. J Dtsch Dermatol Ges. 2010;8(6):411-26. https://doi.org/ 10.1111/j.1610-0387.2010.07339.x.

5. Gisondi P, Cazzaniga S, Chimenti S, Maccarone M, Picardo M, Girolomoni G, et al. Latent tuberculosis infection in patients with chronic plaque psoriasis: evidence from the Italian Psocare Registry. Br J Dermatol. 2015;172(6):1613-20. https://doi.org/10.1111/ bjd.13539.

6. Snast I, Atzmony L, Braun M, Hodak E, Pavlovsky L. Risk for hepatitis $\mathrm{B}$ and $\mathrm{C}$ virus reactivation in patients with psoriasis on biologic therapies: a retrospective cohort study and systematic review of the literature. J Am Acad Dermatol. 2017;77(1):8897e5. https://doi.org/10.1016/j.jaad.2017.01.037.

7. Reich K, Mrowietz U, Radtke MA, Thaci D, Rustenbach SJ, Spehr C, et al. Drug safety of systemic treatments for psoriasis: results from The German Psoriasis Registry PsoBest. Arch Dermatol Res. 2015;307(10):875-83. https://doi.org/10.1007/s0040 3-015-1593-8.

8. Smolen JS, Landewe RBM, Bijlsma JWJ, Burmester GR, Dougados M, Kerschbaumer A, et al. EULAR recommendations for the management of rheumatoid arthritis with synthetic and biological disease-modifying antirheumatic drugs: 2019 update. Ann Rheum Dis. 2020;79(6):685-99. https://doi.org/10.1136/annrheumdi s-2019-216655.

9. FDA. Highlights of prescribing information (Alefacept). https:// www.accessdata.fda.gov/drugsatfda_docs/label/2012/125036s014 4lbl.pdf. Accessed 24 Sept 2020.

10. Jenneck C, Novak N. The safety and efficacy of alefacept in the treatment of chronic plaque psoriasis. Ther Clin Risk Manag. 2007;3(3):411-20.

11. Munn Z, Peters MDJ, Stern C, Tufanaru C, McArthur A, Aromataris E. Systematic review or scoping review? Guidance for authors when choosing between a systematic or scoping review approach. BMC Med Res Methodol. 2018;18(1):143. https://doi. org/10.1186/s12874-018-0611-x.

12. Atalay $\mathrm{S}$, van den Reek J, den Broeder AA, van Vugt LJ, Otero $\mathrm{ME}, \mathrm{Njoo} \mathrm{MD}$, et al. Comparison of tightly controlled dose reduction of biologics with usual care for patients with psoriasis: a randomized clinical trial. JAMA Dermatol. 2020. https://doi. org/10.1001/jamadermatol.2019.4897.

13. Blauvelt A, Ferris LK, Yamauchi PS, Qureshi A, Leonardi CL, Farahi K, et al. Extension of ustekinumab maintenance dosing interval in moderate-to-severe psoriasis: results of a phase IIIb, randomized, double-blinded, active-controlled, multicentre study (PSTELLAR). Br J Dermatol. 2017;177(6):1552-61. https://doi. org/10.1111/bjd.15722.

14. Lebwohl M, Strober B, Menter A, Gordon K, Weglowska J, Puig L, et al. Phase 3 studies comparing brodalumab with ustekinumab in psoriasis. N Engl J Med. 2015;373(14):1318-28. https://doi. org/10.1056/NEJMoa1503824.

15. Reich K, Puig L, Szepietowski JC, Paul C, Lacour JP, Tsianakas A, et al. Secukinumab dosing optimization in patients with 
moderate to severe plaque psoriasis: results from the randomised, open-label OPTIMISE study. Br J Dermatol. 2019. https://doi. org/10.1111/bjd.18143.

16. van Bezooijen JS, van Doorn MBA, Schreurs MWJ, Koch BCP, Te Velthuis H, Prens EP, et al. Prolongation of biologic dosing intervals in patients with stable psoriasis: a feasibility study. Ther Drug Monit. 2017;39(4):379-86. https://doi.org/10.1097/ ftd. 0000000000000420 .

17. Ovejero-Benito MC, Munoz-Aceituno E, Sabador D, Reolid A, Llamas-Velasco M, Prieto-Perez R, et al. Polymorphisms associated with optimization of biological therapy through drug dose reduction in moderate-to-severe psoriasis. J Eur Acad Dermatol Venereol. 2020. https://doi.org/10.1111/jdv.16256.

18. Taniguchi T, Noda S, Takahashi N, Yoshimura H, Mizuno K, Adachi M. An observational, prospective study of monthly adalimumab therapy for disease maintenance in psoriasis patients: a possible new therapeutic option for good responders to the initial induction treatment. J Eur Acad Dermatol Venereol. 2013;27(11):1444-7. https://doi.org/10.111 $1 / \mathrm{j} .1468-3083.2012 .04610 . x$.

19. Baniandres O, Rodriguez-Soria VJ, Romero-Jimenez RM, Suarez R. Dose modification in biologic therapy for moderate to severe psoriasis: a descriptive analysis in a clinical practice setting. Actas Dermosifiliogr. 2015;106(7):569-77. https://doi.org/10.1016/j. ad.2015.02.003.

20. Bardazzi F, Loi C, Prignano F, Ricceri F, Giordano F, Patrizi A, et al. Down-titration of infliximab: the real-life use in psoriatic patients. J Drugs Dermatol. 2016;15(12):1584-6.

21. Carrascosa JM, Garcia-Doval I, Perez-Zafrilla B, Carretero G, Vanaclocha F, Dauden E, et al. Use of off-label doses is frequent in biologic therapy for moderate to severe psoriasis: a cross-sectional study in clinical practice. J Dermatolog Treat. 2015;26(6):502-6. https://doi.org/10.3109/09546634.2015.1034070.

22. Esposito M, Gisondi P, Conti A, Giunta A, Del Giglio M, Di Mercurio M, et al. Dose adjustment of biologic therapies for psoriasis in dermatological practice: a retrospective study. J Eur Acad Dermatol Venereol. 2017;31(5):863-9. https://doi.org/10.1111/ jdv.14145.

23. Fotiadou C, Lazaridou E, Sotiriou E, Ioannides D. Adalimumab for psoriasis in Greece: clinical experience in a tertiary referral centre. J Eur Acad Dermatol Venereol. 2012;26(10):1298-303. https://doi.org/10.1111/j.1468-3083.2011.04290.x.

24. Hansel K, Bianchi L, Lanza F, Bini V, Stingeni L. Adalimumab dose tapering in psoriasis: predictive factors for maintenance of complete clearance. Acta Derm Venereol. 2017;97(3):346-50. https://doi.org/10.2340/00015555-2571.

25. Lee EB, Thomas LW, Egeberg A, Wu JJ. Dosage adjustments in patients with psoriasis on adalimumab-a retrospective chart review. J Eur Acad Dermatol Venereol. 2018;32(7):e292-3. https ://doi.org/10.1111/jdv.14826.

26. López-Ferrer A, Vilarrasa E, Gich IJ, Puig L. Adalimumab for the treatment of psoriasis in real life: a retrospective cohort of 119 patients at a single Spanish centre. Br J Dermatol. 2013;169(5):1141-7. https://doi.org/10.1111/bjd.12543.

27. Piaserico S, Gisondi P, De Simone C, Marinello E, Conti A, Amerio P, et al. Down-titration of adalimumab and etanercept in psoriatic patients: a multicentre observational study. Acta Derm Venereol. 2016;96(2):251-2. https://doi.org/10.2340/00015 555-2209.

28. Puig L, Morales-Múnera CE. Implementing therapeutic goals in maintenance treatment of psoriasis with biologics: adalimumab can be more cost-effective than infliximab or ustekinumab in real life. Eur J Dermatol. 2013;23(3):407-8. https://doi.org/10.1684/ ejd.2013.2017.

29. Romero-Jimenez RM, Escudero-Vilaplana V, Baniandres Rodriguez O, Garcia-Gonzalez X, Sanjurjo SM. Efficiency of biological therapies in patients with moderate to severe psoriasis: impact of a pharmacotherapeutic protocol. J Dermatolog Treat. 2016;27(3):198-202. https://doi.org/10.3109/09546 634.2015.1088127.

30. Romero-Jimenez RM, Escudero-Vilaplana V, Baniandres Rodriguez O, Garcia Martin E, Mateos Mayo A, Sanjurjo SM. Association between clinical factors and dose modification strategies in the treatment with ustekinumab for moderate-to-severe plaque psoriasis. J Dermatolog Treat. 2018;29(8):792-6. https://doi. org/10.1080/09546634.2018.1466978.

31. Feldman SR, Krueger GG. Psoriasis assessment tools in clinical trials. Ann Rheum Dis. 2005;64(Suppl 2):ii65-68; discussion ii973. https://doi.org/10.1136/ard.2004.031237.

32. Carretero G, Puig L, Carrascosa JM, Ferrandiz L, Ruiz-Villaverde $\mathrm{R}$, de la Cueva $\mathrm{P}$, et al. Redefining the therapeutic objective in psoriatic patients candidates for biological therapy. J Dermatolog Treat. 2018;29(4):334-46. https://doi.org/10.1080/09546 634.2017.1395794.

33. Verhoef LM, Selten EMH, Vriezekolk JE, de Jong AJL, van den Hoogen FHJ, den Broeder AA, et al. The patient perspective on biologic DMARD dose reduction in rheumatoid arthritis: a mixed methods study. Rheumatology (Oxford). 2018;57(11):1947-55. https://doi.org/10.1093/rheumatology/key205.

34. Smolen JS, Landewe R, Bijlsma J, Burmester G, Chatzidionysiou $\mathrm{K}$, Dougados M, et al. EULAR recommendations for the management of rheumatoid arthritis with synthetic and biological diseasemodifying antirheumatic drugs: 2016 update. Ann Rheum Dis. 2017;76(6):960-77. https://doi.org/10.1136/annrheumdis-2016210715.

35. Ye W, Tucker LJ, Coates LC. Tapering and discontinuation of biologics in patients with psoriatic arthritis with low disease activity. Drugs. 2018;78(16):1705-15. https://doi.org/10.1007/s4026 5-018-0994-3.

36. Hsu L, Snodgrass BT, Armstrong AW. Antidrug antibodies in psoriasis: a systematic review. Br J Dermatol. 2014;170(2):261-73. https://doi.org/10.1111/bjd.12654.

37. Strand V, Balsa A, Al-Saleh J, Barile-Fabris L, Horiuchi T, Takeuchi $\mathrm{T}$, et al. Immunogenicity of biologics in chronic inflammatory diseases: a systematic review. BioDrugs. 2017;31(4):299_ 316. https://doi.org/10.1007/s40259-017-0231-8.

38. Reich K, Wozel G, Zheng H, van Hoogstraten HJ, Flint L, Barker J. Efficacy and safety of infliximab as continuous or intermittent therapy in patients with moderate-to-severe plaque psoriasis: results of a randomized, long-term extension trial (RESTORE2). Br J Dermatol. 2013;168(6):1325-34. https://doi.org/10.1111/ bjd.12404.

39. Higgins. JPT. Methodological Expectations of Cochrane Intervention Reviews. Cochrane, London. 2019. https://community.cochr ane.org/sites/default/files/uploads/inline-files/MECIR\%20Oct ober\%202019\%20Final\%20Online\%20version.pdf. Accessed 24 Sep 2020.

40. Na JI, Kim JH, Park KC, Youn SW. Low-dose etanercept therapy in moderate to severe psoriasis in Korean. J Dermatol. 2008;35(8):484-90. https://doi.org/10.111 $1 / \mathrm{j} .1346-8138.2008 .00508 . x$. 


\section{Affiliations}

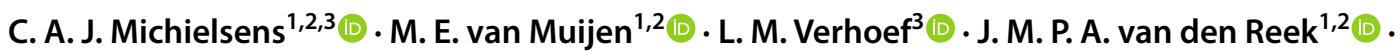
E. M. G. J de Jong 1,2,4 $^{1}$

1 Radboud University Medical Center, Radboud Institute for Health Sciences, Mailbox 9101, 6500 HB Nijmegen, The Netherlands

2 Department of Dermatology, Radboud University Medical Center, Nijmegen, The Netherlands
3 Department of Rheumatology, Sint Maartenskliniek, Ubbergen, The Netherlands

4 Radboud University, Nijmegen, The Netherlands 